\title{
Revision surgery for chronically discharging mastoid cavities: mastoid obliteration with canal wall reconstruction versus non-obliteration surgery
}

\author{
Hylke F. E. van der Toom ${ }^{1}$ (D) Marc P. van der Schroeff ${ }^{1} \cdot$ Tim L. Molenaar $^{1} \cdot$ Mick Metselaar $^{1} \cdot$ Anne van Linge $^{1}$. \\ Jantien L. Vroegop ${ }^{1} \cdot$ Robert J. Pauw ${ }^{1}$
}

Received: 31 August 2021 / Accepted: 12 October 2021 / Published online: 27 October 2021

(c) The Author(s) 2021

\begin{abstract}
Purpose To evaluate the surgical results of revision canal wall down (CWD) surgery for chronically discharging mastoid cavities and to compare the non-obliteration approach to mastoid obliteration with canal wall reconstruction.

Methods This is a retrospective cohort study. All adult patients ( $\geq 18$ years) who underwent revision surgery for chronically draining mastoid cavities between January 2013 and January 2020 were included. Primary outcome measures included the dry ear rate, complications and postoperative hearing.

Results 79 ears were included; 56 ears received revision CWD with mastoid obliteration and posterior canal wall reconstruction and 23 ears received CWD without mastoid obliteration. The dry ear rate at the most recent outpatient clinic visit (median 28.0 months postoperative) was significantly higher in the obliteration group with $96.4 \%$ compared to $73.9 \%$ for the non-obliteration group $(p=.002)$. There were no differences in audiological outcome and incidence of complications between the two techniques.

Conclusion We show that in our study population revision CWD surgery with mastoid obliteration and posterior canal wall reconstruction is superior to revision CWD surgery without mastoid obliteration in the management of chronically discharging mastoid cavities. In the obliteration group, a dry ear was achieved in $96.4 \%$ as this was $73.9 \%$ in the non-obliteration group. We found no differences in audiological outcome and in incidence of complications between the two techniques.
\end{abstract}

Keywords Mastoid cavity $\cdot$ Mastoid bowl $\cdot$ Revision $\cdot$ Obliteration $\cdot$ Otitis $\cdot$ Cholesteatoma

\section{Introduction}

While obliteration techniques in the surgical management of cholesteatoma gain popularity [1], cholesteatoma surgery is still often performed using the traditional canal wall up (CWU) or canal wall down (CWD) approach without obliteration. Although the recurrent and residual cholesteatoma rates after CWD without obliteration are lower compared to CWU without obliteration [2, 3], the CWD technique carries several disadvantages caused by the loss of self-cleaning capacity of the ear such as chronical discharge and infection

Hylke F. E. van der Toom

h.vandertoom@erasmusmc.nl

1 Department of Otorhinolaryngology and Head and Neck Surgery, Erasmus Medical Center, Doctor Molewaterplein 40, 3015 GD Rotterdam, The Netherlands of the persistent mastoid bowl, need for regular maintenance cleaning at the outpatient clinic, caloric-induced dizziness after temperature or pressure changes, the need for precautionary water avoidance measures and difficulty to fit hearing aids [4-8]. Reasons for surgical failure after CWD surgery include residual infected mastoid air cells, large mastoid cavities, high facial ridge, bony overhang in the mastoid cavity, tympanic membrane perforations, narrow meatus and cholesteatoma [9-11]. Discharging mastoid bowls are usually successfully controlled by frequent maintenance cleaning and topical therapy. However, in a small portion of patients, intensive topical treatment does not lead to disease control. In such cases, surgical revision may be necessary to achieve a stable ear. Several surgical techniques have been described in the management of the chronically discharging mastoid cavity, including revision canal wall down mastoidectomy with or without mastoid obliteration and with or without reconstruction of the posterior canal wall [4, 8-17]. 
The main goal of these interventions is to achieve the highest possible postoperative long-term dry ear rate. While the aforementioned studies have reported results using either obliteration or non-obliteration techniques alone, there is to our knowledge no study that compares mastoid obliteration with canal wall reconstruction to non-obliteration surgery in the management of chronically discharging mastoid cavities. Therefore, it would be interesting to compare the two surgical techniques with regards to the postoperative dry ear rate, complications and postoperative hearing.

In our tertiary referral center, obliteration of the mastoid with posterior canal wall reconstruction for the management of chronically discharging mastoid bowls was introduced in 2013 and gradually replaced the revision canal wall down mastoidectomy without obliteration and canal wall reconstruction. As these different approaches were performed by the same otologic surgeons, this cohort lends well for comparison of the surgical outcome of the two techniques. Therefore, the aim of this study is to evaluate the surgical results of revision surgery for chronically discharging mastoid cavities and to compare the non-obliteration approach to mastoid obliteration with posterior canal wall reconstruction.

\section{Materials and methods}

\section{Patients}

A retrospective cohort study was performed at our department with approval of the medical research ethical committee. All adult patients ( $\geq 18$ years) who underwent revision surgery for chronically draining mastoid cavities between January 2013 and January 2020 were included. All patients were previously adequately but unsuccessfully treated with topical therapy. The following variables were retrieved from electronic patient records: patient characteristics, indication for surgery (chronically infection, recurrent or residual cholesteatoma, bothersome maintenance cleaning), preoperative symptoms (discharge, pain, vertigo), tympanic membrane status, date of surgery, surgeon, whether or not mastoid obliteration with posterior canal wall reconstruction was performed, reasons for previous surgical failure (high facial ridge, residual mastoid cells, bony overhang, cholesteatoma, tympanic membrane perforation, narrow meatus), administration of antibiotics perioperatively, ossicular chain status (intact chain, absent incus, absent stapes, fixated footplate), ossicular chain reconstruction (no reconstruction due to intact chain, no reconstruction but damaged chain, incus interposition, partial ossicular replacement prosthesis (PORP), total ossicular replacement prosthesis (TORP), tympanic membrane to stapes (type III reconstruction), tympanic membrane directly to footplate), pre- and postoperative hearing (air conduction (AC), bone conduction
(BC) and air-bone gap (ABG) for 0.5, 1, 2 and $4 \mathrm{kHz}$ ), postoperative symptoms (discharge, pain, vertigo), postoperative frequency of maintenance cleaning at outpatient clinic, postoperative otoscopy at the outpatient clinic at different timepoints. The postoperative otoscopy was scored as 1) fully dry, 2) minimal discharge or 3) infection.

\section{Surgical technique}

The surgical technique for mastoid obliteration with posterior canal wall reconstruction was performed as described by Vercruysse et al [8]. Surgery was performed under general anesthesia. In case of obliteration, two grams of cefazolin was administrated intravenously prior to surgery and eventually repeated after $4 \mathrm{~h}$. If necessary, a M-meatoplasty [18] was performed. After a wide retro-auricular question markshaped incision, the mastoid bowl was exposed and granulation tissue was removed. Remaining mastoid cells were removed and in case of obliteration and posterior canal wall reconstruction, cortical bone dust and cortical bone chips or conchal cartilage were collected and stored in a rifamycin solution (Sanofi-Aventis U.S. LLC, Bridgewater, New Jersey, USA. Rifadin ${ }^{\circledR} 600 \mathrm{mg}$ powder for infusion with $10 \mathrm{~mL}$ solvent for solution and $20 \mathrm{~mL} 0.9 \%$ sodium chloride). After removal of all accessible cell tracts, possible bony overhang, possible cholesteatoma and/or after lowering the facial ridge, in case of mastoid obliteration, the posterior canal wall was reconstructed with cartilage or bone chips and covered with temporal fascia or a mid-temporal artery flap followed by mastoid obliteration with bone dust upon the level of the mastoid cortex. When no sufficient autologous bone dust was available for obliteration, bioactive glass granules (Bonealive ${ }^{\circledR}$, Bonalive Biomaterials Ltd., Turku, Finland) were used. In most cases when no obliteration was performed, the mastoid was smoothened with some bone dust. In these cases, a mastoid cavity persisted. If possible an ossicular chain reconstruction was performed and if necessary the tympanic membrane was reconstructed with cartilage or temporal fascia. The ear canal was packed with a gauze with hydrocortisone/oxytetracycline/polymyxin B for at least 1 week and was usually re-packed after 1 week.

\section{Statistical analyses}

Statistical analyses were performed using IBM SPSS Statistics 26 (SPSS Inc., Chicago, IL, USA) and Graphpad Prism 7.0 (GraphPad Software, La Jolla California, USA). The Chi-square test of independence was performed to examine for differences in surgical outcome between the surgical techniques. For hearing outcome and data on time intervals for postoperative maintenance cleaning (not normally distributed), the Mann-Whitney $U$ test was used to assess 
differences between the surgical techniques. $P$-values $<0.05$ were considered statistically significant.

\section{Results}

\section{Patients}

Between January 2013 and January 2020, 79 ears of 76 patients received revision surgery due to a chronically draining mastoid cavity. The median follow-up time was 28 months (inter quartile range (IQR) 17-55 months). Preoperatively, 78 patients $(98.7 \%)$ suffered from chronic discharge, 11 patients $(13.9 \%)$ from vertigo, 6 patients $(7.6 \%)$ from pain and 1 patient $(1.3 \%)$ from bothersome recurrent cleaning. Obliteration of the mastoid with posterior canal wall reconstruction was performed in 56 cases $(70.9 \%)$ whilst no obliteration was performed in 23 cases $(29.1 \%)$. The most frequently used obliteration material was autologous bone dust ( $80.4 \%$ ), followed by autologous bone dust

Table 1 Reasons for previous surgical failure

\begin{tabular}{lc}
\hline Reason & $N(\%)$ \\
\hline Remaining mastoid air cells with infected mucosa & $73(92.4 \%)$ \\
High facial ridge & $37(46.8 \%)$ \\
Narrow meatus & $27(34.2 \%)$ \\
Remaining bony overhang & $25(31.6 \%)$ \\
Cholesteatoma & $14(17.7 \%)$ \\
Tympanic membrane perforation & $13(16.5 \%)$ \\
Exposed bone in mastoid bowl & $3(3.8 \%)$ \\
\hline
\end{tabular}

together with bioactive glass granules (16.1\%) and bioactive glass granules alone (3.6\%). The posterior canal wall was reconstructed with bone chips in $71.4 \%$, cartilage in $12.5 \%$ and bone chips with cartilage in $16.1 \%$. Patient characteristics are shown in supplemental Table 1.

\section{Reasons for previous surgical failure}

The reasons for previous surgical failure are shown in Table 1. Remaining mastoid air cells with infected mucosa contributed to the chronic discharge in $92.4 \%$ of cases, a high facial ridge in $46.8 \%$ of cases, a narrow meatus in $34.2 \%$ of cases, a remaining bony overhang in $31.6 \%$ of cases, cholesteatoma in $17.7 \%$ of cases, a tympanic membrane perforation in $16.5 \%$ of cases and exposed bone in the mastoid bowl in $3.8 \%$ of cases.

\section{Surgical outcome}

The surgical outcome per technique is shown in Table 2 . The dry ear rate at 8 weeks postoperatively, 1 year postoperatively and at the most recent outpatient clinic visit (median 28.0 months postoperative) was 57.1, 91.1 and $96.4 \%$, respectively for the obliteration group and $60.9,65.2$ and $73.9 \%$, respectively for the non-obliteration group. At 1 year postoperatively and at the most recent examination, the dry ear rate was significantly higher in the obliteration group compared to the non-obliteration group ( $p=0.007$ and $p=0.002$, respectively). At most recent follow-up, vertigo and pain was not present in any cases in the obliteration and non-obliteration group. There was persistent need for regular maintenance cleaning in $82.1 \%$ of patients with mastoid obliteration and $100 \%$ in the non-obliteration group
Table 2 Surgical outcome per technique

\begin{tabular}{lllll}
\hline & & $\begin{array}{l}\text { Obliteration with canal } \\
\text { wall reconstruction }\end{array}$ & No obliteration & $P$ \\
\hline Number of cases & & 56 & 23 & \\
Dry ear 8 weeks postoperatively & Yes & $32(57.1 \%)$ & $9(39.1 \%)$ & 0.145 \\
& No & $24(42.9 \%)$ & $14(60.9 \%)$ & \\
Dry ear 1 year postoperatively & Yes & $51(91.1 \%)$ & $15(65.2 \%)$ & $\mathbf{0 . 0 0 7}$ \\
& No & $5(8.9 \%)$ & $8(34.8 \%)$ & \\
Dry ear at last visit & Yes & $54(96.4 \%)$ & $17(73.9 \%)$ & $\mathbf{0 . 0 0 1}$ \\
& No & $2(3.6 \%)$ & $6(26.1 \%)$ & \\
Need for regular maintenance & Yes & $46(82.1 \%)$ & $23(100 \%)$ & $\mathbf{0 . 0 3 9}$ \\
cleaning & No & $9(16.1 \%)$ & $0(0 \%)$ & 0.417 \\
Need for revision surgery & Yes & $8(14.3 \%)$ & $19(82.6 \%)$ & \\
\multirow{2}{*}{$\begin{array}{l}\text { Median interval for maintenance cleaning in } \\
\text { months (IQR) }\end{array}$} & No & $48(85.7 \%)$ & $6.0(4.0-10.5)$ & $\mathbf{0 . 0 0 5}$ \\
\hline
\end{tabular}

Bold values indicate a significant difference $(P$-value $<0.05)$ between the obliteration and non-obliteration group 
Table 3 Reasons for revision surgery

\begin{tabular}{ll}
\hline Reasons & $N(\%)$ \\
\hline Revision CWD due to cholesteatoma & $3(25 \%)$ \\
Revision CWD due to exposed bone & $2(16.7 \%)$ \\
Meatoplasty & $2(16.7 \%)$ \\
Remaining mastoid air cells with infected mucosa & $1(8.3 \%)$ \\
Revision ossicular chain & $1(8.3 \%)$ \\
Excision subcutaneous cholesteatoma local anesthesia & $1(8.3 \%)$ \\
Subtotal petrosectomy & $1(8.3 \%)$ \\
Liquorrhea from posterior cranial fossa & $1(8.3)$ \\
Total & 12 \\
\hline
\end{tabular}

CWD canal wall down

$(p=0.039)$ and the median time interval for maintenance cleaning at the outpatient clinic was significantly longer in the obliteration group compared to the non-obliteration group (12.0 months versus 6.0 months, $p=0.005$ ).

\section{Revision surgery and cholesteatoma recurrence}

To achieve a dry ear, revision surgery was needed in 8 cases $(14.3 \%)$ in the obliteration group and in 4 cases (17.4\%) in the non-obliteration group (Table 3), mostly because of recurrent or residual cholesteatoma ( 3 cases, $25.0 \%$ of revisions), exposed bone ( 2 cases, $16.7 \%$ of revisions) or a narrow meatus ( 2 cases, $16.7 \%$ of revisions). In 1 case after revision CWD without obliteration, a subtotal petrosectomy was performed because of persistent discharge and fear for maintenance cleaning.

During follow-up of the 14 cholesteatoma cases, a residual cholesteatoma was detected in 1 case (subcutaneously at incision site, detected at 76 months postoperatively) and a recurrent cholesteatoma in 2 cases (detected after 31 and 32 months postoperatively, respectively), resulting in a residual cholesteatoma rate of $7.1 \%$ and a recurrent cholesteatoma rate of $14.3 \%$.

\section{Postoperative hearing}

As shown in supplemental Table 2, there were no significant differences in perioperative ossicular chain status between the two groups. For ossicular chain reconstruction, there were no significant differences between the two groups besides for type III ossicular chain reconstruction which was more often performed in the non-obliteration group compared to the obliteration group $(p=0.022)$.

Postoperative hearing tests were available for 52 patients (92.8\%) in the obliteration group and $21(91.3 \%)$ patients in the non-obliteration group (Table 4). There were no significant differences in pre-, postoperative and change in $\mathrm{AC}$

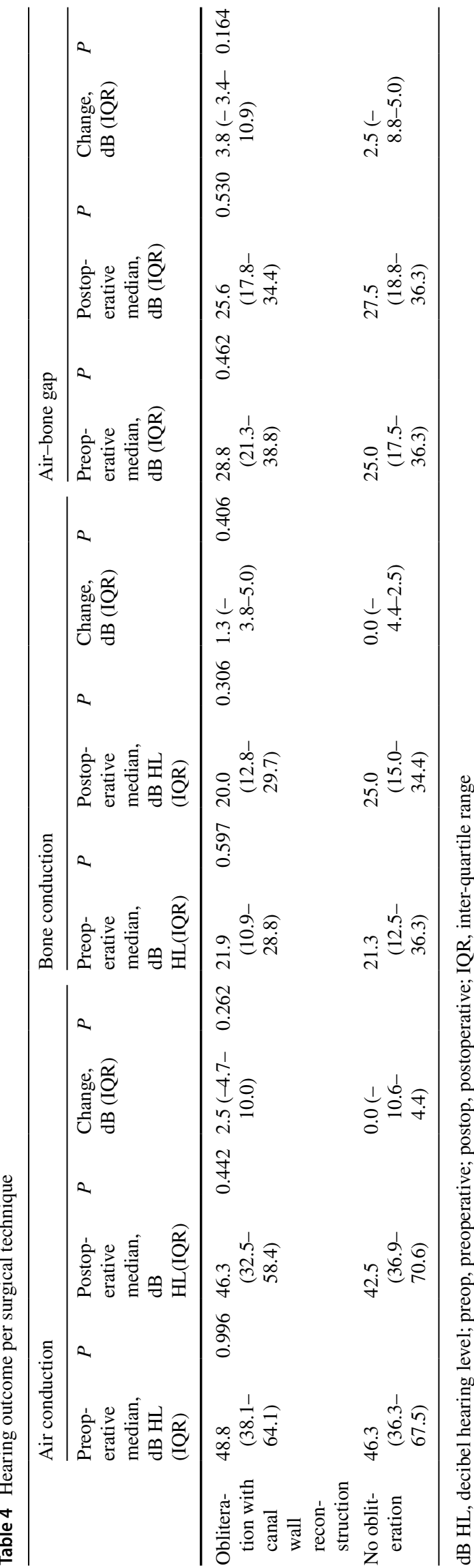


threshold, $\mathrm{BC}$ threshold and $\mathrm{ABG}$ between the two groups. The median postoperative AC threshold was $46.3 \mathrm{~dB}$ HL in the obliteration group and $42.5 \mathrm{~dB}$ HL in the non-obliteration group. There were no cases of iatrogenic sensorineural hearing loss.

\section{Complications}

A postoperative otitis externa within 3 months post-surgery was observed in 3 cases $(5.4 \%)$ in the obliteration group and in 4 cases (17.4\%) in the non-obliteration group. Six infections were successfully controlled with topical therapy as in 1 case oral antibiotics were administered. In 2 cases (3.6\%) in the obliteration group, liquorrhea occurred due to an iatrogenic defect in the cranial fossa. In 1 case, this was successfully closed during the initial procedure and in 1 case revision surgery was needed to close a defect in the posterior cranial fossa.

\section{Literature overview}

The current literature on revision mastoidectomy for troublesome cavities is relatively scarce, as shown in a literature overview in Table 5 . The dry ear rates after revision CWD range from 6-98\% for the non-obliteration techniques and $40-100 \%$ for the obliteration techniques. However, it is hard to compare the different studies due to differences in sample size, follow-up duration, surgical technique and obliteration material and if whether or not a posterior canal wall reconstruction was performed.

\section{Discussion}

In this study, we retrospectively compared revision canal wall down surgery with mastoid obliteration and posterior canal wall reconstruction to revision canal wall down surgery without obliteration in the management of chronically discharging mastoid cavities. We show that in our study population, the obliteration technique is superior to the non-obliteration technique with regards to the dry ear rate, need for regular maintenance cleaning and interval between maintenance cleaning sessions. We found no differences in audiological outcome and in incidence of complications between the two techniques.

The incidence of discharging mastoid cavities after canal wall down surgery is estimated at 10-30\% [15]. Whilst usually controllable by topical therapy and frequent maintenance cleaning, a therapy-resistant discharging mastoid cavity can be of great burden to the patient and in such cases revision surgery may be indicated. Goals of revision surgery are to create a dry, safe ear with preservation of hearing and with the lowest possible frequency of necessary outpatient clinic visits after healing of the ear. In the present study, the dry ear rate at 1 -year postoperatively was $91.1 \%$ in the obliteration group and $65.2 \%$ in the non-obliteration group; these percentages were 96.4 and $73.9 \%$, respectively at most recent follow-up (median 28.0 months postoperative). The superior results of the obliteration technique compared to the non-obliteration approach may be explained by the fact that the normal anatomy of the external ear canal is restored after obliteration while in a persistent mastoid bowl, cerumen and debris can still accumulate and potentially cause infection. While $82.1 \%$ of patients in the obliteration group still received regular maintenance cleaning at the outpatient clinic postoperatively, the median interval for maintenance cleaning was 12 months in the obliteration group, significantly longer compared to the median 6 months in the nonobliteration group.

As previous studies reported outcome on either the obliteration $[4,8-10,12-14,16,17]$ or the non-obliteration $[10-12,15]$ technique alone, it remained unclear whether the outcome of these studies was a result of the surgical technique itself or a result of the surgeons' skills. As in the present study, the two surgical techniques were performed by the same surgeons in the same institute, we show for the first time that it may actually be the obliteration technique that is robust and an important determinant factor for surgical outcome. In literature (Table 5), dry ear rates varying from 6 to $100 \%$ are reported. Due to differences in surgical technique, obliteration material (bone dust, hydroxyapatite granules, bioactive glass granules, temporal muscle flaps, Palva flaps and mid-temporal artery flaps) and follow-up period it is difficult if not impossible to compare the different studies. However, the studies in which a mastoid obliteration is performed tend to show higher postoperative dry ear rates compared to the non-obliteration studies. It is not possible to investigate the role of the posterior canal wall reconstruction on the dry ear rate in the present literature, and our study design does also not allow us to assess its role on the dry ear rate. However, we believe that a firm posterior canal wall reconstruction prevents the bone pate from dissolving and thus is an important pillar in the surgical management of the chronically discharging mastoid cavity.

We identified the reasons for previous surgical failure to be remaining mastoid air cells with infected mucosa, a high facial ridge, a narrow meatus, remaining bony overhang, cholesteatoma, a tympanic membrane perforation or exposed bone in the mastoid bowl. These findings confirm previous research in which the same factors were identified [9-11] and any otologist performing canal wall down surgery should be aware of the importance in addressing these factors to prevent surgical failure. The postoperative complications consisted mainly of infection of the external ear canal or mastoid bowl, which was observed in 3 cases (5.4\%) in the obliteration group and in 4 cases (17.4\%) in the 


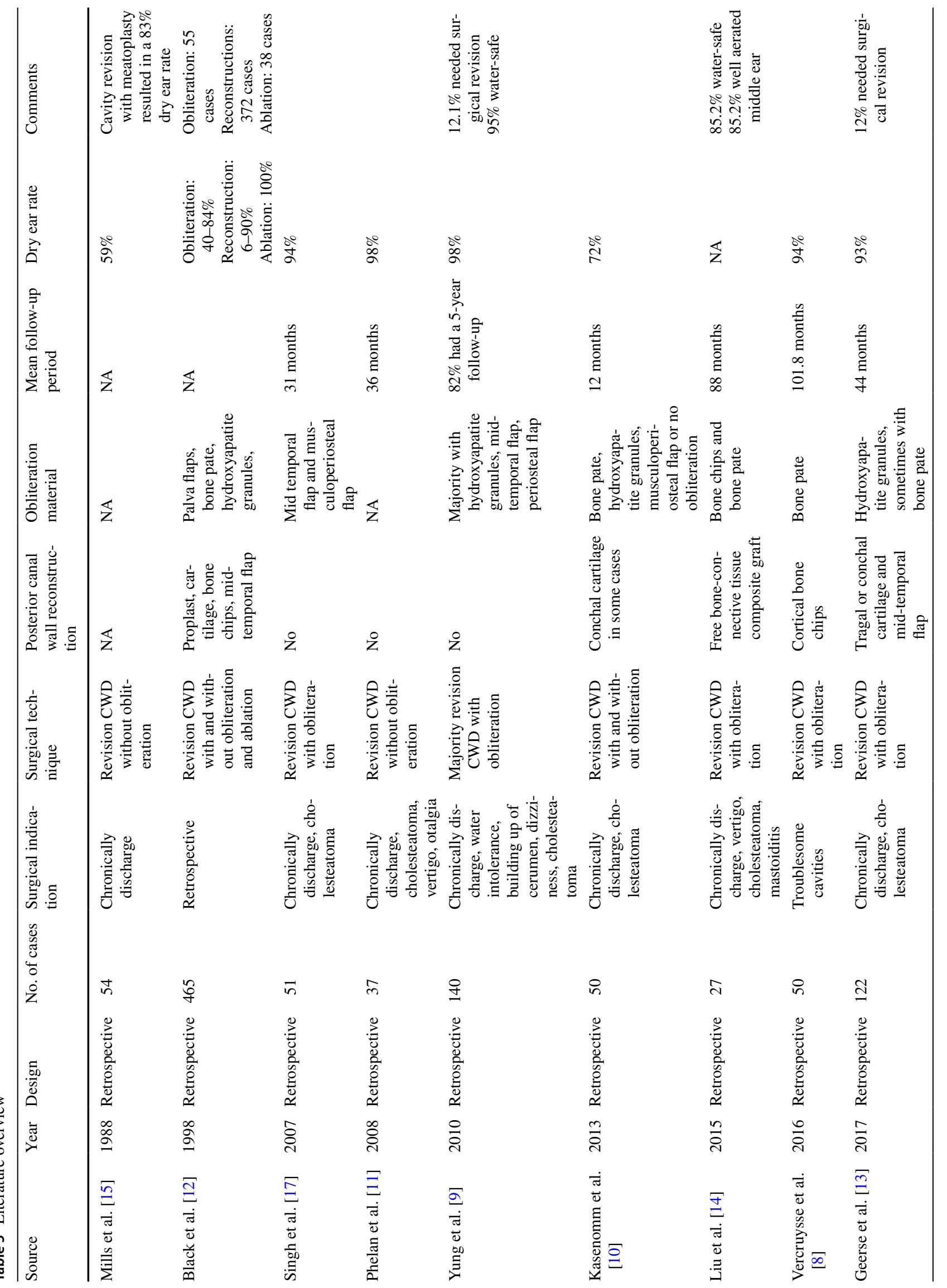




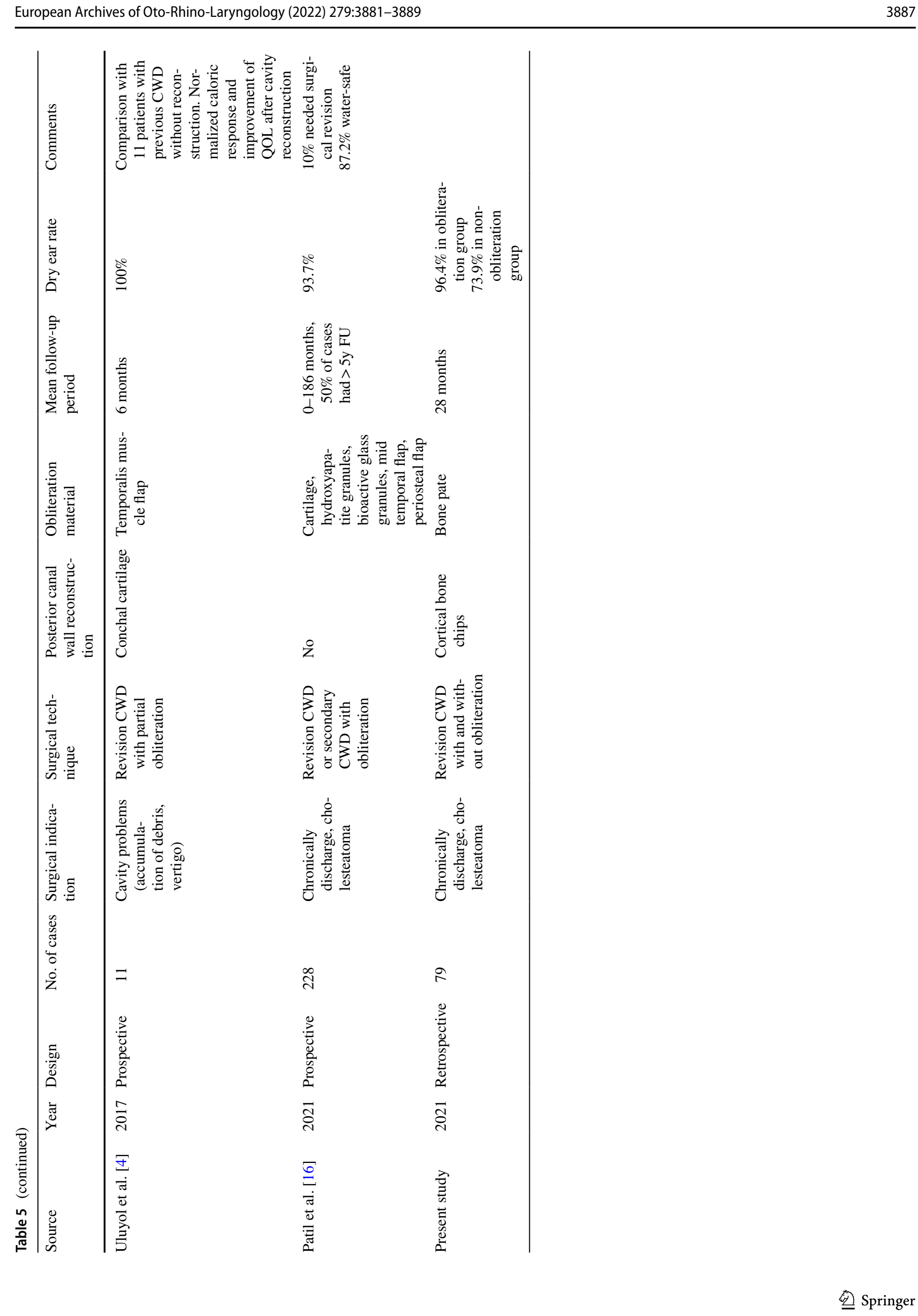


non-obliteration group. However, these were mild infections which were successfully treated with topical antibiotics in 6 cases and with oral antibiotics in 1 case. The relatively high infection rate in the non-obliteration group may be overestimated due to small sample size. The re-intervention rate was $14.3 \%$ in the obliteration group and $17.4 \%$ in the non-obliteration group. These rates are in line with literature in which re-operation rates ranging from 10 to $12.1 \%$ are reported $[9,13,16]$. To prevent revision surgery in the future, we are now aware to always perform a meatoplasty when necessary.

There were no significant differences in ossicular chain status and in postoperative hearing between the two groups. With a median postoperative AC threshold level of $46.3 \mathrm{~dB}$ $\mathrm{HL}$ in the obliteration group and $42.5 \mathrm{~dB}$ HL in the nonobliteration group, the hearing results are to some degree disappointing. However, when interpreting these results one should take into account that the cohort consisted of very challenging chronically discharging ears of which most had several surgical interventions before. Therefore, the aim was to preserve hearing and not necessarily to improve hearing, which was achieved with a median change in AC threshold of $2.5 \mathrm{~dB}$ HL in the obliteration group and $0.0 \mathrm{~dB} \mathrm{HL}$ in the non-obliteration group. Especially patients in the obliteration group should be able to wear hearing devices without any problems because of the restored anatomy. Unfortunately, it was not systematically reported in the patient records whether patients were able to.

\section{Limitations}

Limitations of this study include the retrospective design and the possible indication bias. The mastoid obliteration with posterior canal wall reconstruction procedure was introduced in 2013 in our clinic and gradually replaced revision CWD without obliteration. Especially in the beginning of this transition, the choice to perform one of the two techniques was made by the surgeon based on preferences and experience. Later on, all surgeons adopted the obliteration technique and in principle no non-obliteration techniques were used anymore. Further limitations include the follow-up: the median follow-up duration was 20 months for the obliteration group and 67 months for the non-obliteration group. A shorter follow-up period may potentially overestimate the dry ear rate as long-term failures may not be included. However, no long-term failure was observed in the non-obliteration group and previous research has shown excellent long-term results after the obliteration technique $[9,13]$.

\section{Conclusion}

We show that in our study population revision CWD surgery with mastoid obliteration and posterior canal wall reconstruction is superior to revision CWD surgery without mastoid obliteration in the management of chronically discharging mastoid cavities. In the obliteration group, a dry ear was achieved in $96.4 \%$ as this was $73.9 \%$ in the non-obliteration group. We found no differences in audiological outcome and in incidence of complications between the two techniques.

Supplementary Information The online version contains supplementary material available at https://doi.org/10.1007/s00405-021-07138-0.

Funding None were declared.

\section{Declarations}

Conflict of Interest None were declared.

Open Access This article is licensed under a Creative Commons Attribution 4.0 International License, which permits use, sharing, adaptation, distribution and reproduction in any medium or format, as long as you give appropriate credit to the original author(s) and the source, provide a link to the Creative Commons licence, and indicate if changes were made. The images or other third party material in this article are included in the article's Creative Commons licence, unless indicated otherwise in a credit line to the material. If material is not included in the article's Creative Commons licence and your intended use is not permitted by statutory regulation or exceeds the permitted use, you will need to obtain permission directly from the copyright holder. To view a copy of this licence, visit http://creativecommons.org/licenses/by/4.0/.

\section{References}

1. van der Toom HFE, van der Schroeff MP, Pauw RJ (2018) Singlestage mastoid obliteration in cholesteatoma surgery and recurrent and residual disease rates: a systematic review. JAMA Otolaryngol Head Neck Surg 144(5):440-446

2. Tomlin J, Chang D, McCutcheon B, Harris J (2013) Surgical technique and recurrence in cholesteatoma: a meta-analysis. Audiol Neurotol 18(3):135-142

3. van der Toom H, van der Schroeff MP, Janssen J, Westzaan AM, Pauw RJ (2020) A retrospective analysis and comparison of the STAM and STAMCO classification and EAONO/JOS cholesteatoma staging system in predicting surgical treatment outcomes of middle ear cholesteatoma. Otol Neurotol 41(4):e468-e474

4. Uluyol S, Ugur O, Arslan IB, Yagiz O, Gumussoy M, Cukurova I (2018) Effects of cavity reconstruction on morbidity and quality of life after canal wall down tympanomastoidectomy. Braz J Otorhinolaryngol 84(5):608-613

5. Mehta RP, Harris JP (2006) Mastoid obliteration. Otolaryngol Clin North Am 39(6):1129-1142

6. Palva T (1973) Operative technique in mastoid obliteration. Acta Otolaryngol 75(4):289-290

7. Sade J, Weinberg J, Berco E, Brown M, Halevy A (1982) The marsupialized (radical) mastoid. J Laryngol Otol 96(10):869-875

8. Vercruysse JP, van Dinther JJ, De Foer B et al (2016) Long-term Results of troublesome CWD cavity reconstruction by mastoid 
and epitympanic bony obliteration (CWR-BOT) in adults. Otol Neurotol 37(6):698-703

9. Yung M, Tassone P, Moumoulidis I, Vivekanandan S (2011) Surgical management of troublesome mastoid cavities. J Laryngol Otol 125(3):221-226

10. Kasenomm $P$ (2013) Intraoperative findings of revision canal walldown tympanomastoid surgery. Acta Otolaryngol 133(8):826-832

11. Phelan E, Harney M, Burns $H$ (2008) Intraoperative findings in revision canal wall down mastoidectomy. Ir Med J 101(1):14

12. Black B (1998) Mastoidectomy elimination: obliterate, reconstruct, or ablate? Am J Otol 19(5):551-557

13. Geerse S, Ebbens FA, de Wolf MJ, van Spronsen E (2017) Successful obliteration of troublesome and chronically draining cavities. J Laryngol Otol 131(2):138-143

14. Liu SC, Wang CH, Huang BR (2015) Obliteration of radical cavities and total reconstruction procedure without staging after canal wall down mastoidectomy: long-term results. Clin Exp Otorhinolaryngol 8(3):230-236

15. Mills RP (1988) Surgical management of the discharging mastoid cavity. J Laryngol Otol Suppl 16:1-6

16. Patil S, Trinidade A, Wong B, Yung MW (2021) Secondary obliteration surgery for troublesome mastoid cavities: a prospective series of 228 pediatric and adult cases. Otol Neurotol 42(7):e881-e886

17. Singh V, Atlas M (2007) Obliteration of the persistently discharging mastoid cavity using the middle temporal artery flap. Otolaryngol Head Neck Surg 137(3):433-438

18. Mirck PG (1996) The M-meatoplasty of the external auditory canal. Laryngoscope 106(3 Pt 1):367-369

Publisher's Note Springer Nature remains neutral with regard to jurisdictional claims in published maps and institutional affiliations. 